\title{
USCF ADOPTS OFFICIAL RULES FOR PLAY INVOLVING COMPUTERS
}

During the USCF Convention, the Board of Delegates also adopted a set of official rules for play involving computers, based on the ICCA rules as submitted by Ken Thompson. These new rules read as follows:

\section{RULES FOR PLAY INVOLVING COMPUTERS}

The following rules are for USCF-rated tournaments when one of the players (or both) is a computer. In matters not covered by these rules, play is governed by applicable human rules, as interpreted by the arbiter. In these rules, the term "computer" refers to a Chess program running on a computer. The term "opponent" refers to the computer's opponent, human or computer. The term "operator" refers to the per'son running the computer. The following rules shall govern play:

\section{PARAMETER SETTINGS}

Before play begins, the operator shall do all initial setting up of the computer. At this time, the operator may freely specify any operational parameters, such as rate of play, suggested openings, value of a draw, etc. After play begins, the role of the operator is passive. As such, the operator is not allowed to alter any parameter settings during play that might alter the course of the game.

2. COMMUNICATION OF MOVES

During play, the operator is to communicate the opponent's moves to the computer.

\section{EXECUTION OF MOVES}

The operator is to execute the computer's specified move on the playing chessboard. "Touch" rules do not strictly apply to the operator, although blatant cases may be violations of other rules.

\section{CLOCK}

After the computer's move is executed, the operator is to start the opponent's clock.

\section{RECONCILIATION OF POSITIONS}

If, during play, different positions should arise on the playing chessboard and the computer's representation thereof, due to operator. error, such differences shall be corrected with the assistance of the arbiter. The arbiter may choose either to accept the playing chessboard as official, or to retrace the moves to the point of departure. If the arbiter chooses to back up the game, then the arbiter shall readjust the clocks accordingly.

\section{RESETTING THE COMPUTER}

If, during play, the computer is unable to accept a legal move because of discrepancies, communication trouble, or computer trouble, then the operator may set up the current board position and status, along with clock times. Other parameters set must be the same as those in effect at the start of the game. The clocks are not stopped during the resetting of the computer. 


\section{F L A S H}

The next issue of the ICCA Newsletter will have the complete results of the 13th ACM North American Computer Chess Championship. For the first time in history four programs tied for first place, with three points out of a possible four: BELLE, CRAY-BLITZ, NUCHESS, and CHAOS. Don't miss the next issue to read about this exciting tournament.

\section{USCF Adopts Official Rules for Play Involving Computers (continued from page 3)}

\section{CLOCK TIMES}

The operator may communicate the clock times to the computer only if the computer initiates the request.

8. MEMORY UNIT EXCHANGE

The operator may change or insert memory units when the computer requests this and identifies the unit to be inserted, by description or by generating a coded signal or message with a single predetermined meaning. Diskettes, disk cartridges, tapes, ROM cartridges ("program modules" in commercial machines), and the like are all considered equiva lent forms of memory units.

\section{DRAW OFFERS AND RESIGNATION}

The operator may offer a draw, accept a draw, or resign on behalf of the computer. This may be done with or without computer consultation.

\section{TIME FORFEITS}

The operator may claim the game in cases where the opponent has exceeded his time limit.

\section{ADJOURNMENT}

The operator shall carry out the necessary adjournment formalities.

12. SCQRE

The operator and/or the computer must keep a score of the game. 